$\begin{array}{lllllllllllllllllllll}A & R & T & I & C & U & L & O & D & E\end{array}$ $\begin{array}{lllllllll}R & E & F & L & E & X & I & O & N\end{array}$

\title{
LA EVALUACIÓN COMO HERRAMIENTA DE CONOCIMIENTO FRENTE A LA DESERCIÓN Y LA MARGINALIDAD
}

\author{
EVALUATION AS A TOOL OF KNOWLEDGE AGAINST DESERTION \\ AND MARGINALITY
}
Por: Carlos Enrique Mosquera Mosquera* Ingrid Giovanna Rondón Márquez**

*Miembro de Grupo de Investigación Educación y Desarrollo de la Universidad Cooperativa de Colombia. Docente investigador de la Corporación Universitaria Politécnico Marco Fidel Suárez. Lic., en Filosofía y Religión de la Universidad Católica de Oriente. Magíster en Educación de la Universidad Santo Tomás. Estudiante del Doctorado en Educación de la Universidad Naciona de Rosario, Argentina. E-mail: carlosfilosofo@ hotmail.com

**Profesional en Negocios Internacionales con Énfasis en Marketing Internacional. Especialista en Gerencia Integral de Proyectos. Magíster en Educación de la Universidad Santo Tomás. Doctoranda en Ciencias Humanas con Mención en Educación de la Universidad Nacional De

Rosario en Argentina. Docente Tiempo completo de la Universidad Cooperativa de Colombia, Sede Barrancabermeja, integrante del grupo de investigación Visión contable, económica y solidaria VICES

E-mail: ingridocente@ gmail.com

\begin{abstract}
RESUMEN
El presente artículo de reflexión surge bajo el análisis de la concepción de evolución que se presenta en el currículo y las prácticas evaluativas de los profesores de la Institución Educativo Santo Tomás de Aquino. La metodología que orientó el trabajo fue de corte documental. Las conclusiones fueron abordadas bajo la interpretación del Decreto 1290, el cual evidenció que: 1. Existe una lejanía entre la forma como está organizado el sistema de evaluación institucional frente a los criterios de evaluación; 2. Las prácticas pedagógicas y evaluativas de muchos docentes difieren del enfoque que propone el Decreto 1290, y sumado a esto, difieren del mismo SIE que ellos mismos han construido como institución; 3. En los documentos prescriptos y practicas pedagógicas de los maestros subyace una concepción tecnicista de la evaluación; 4. La evaluación debe ser un instrumento que fomente el conocimiento, mas no la reprobación, deserción, exclusión social y marginalidad.
\end{abstract}

Palabras clave: prácticas evaluativas, deserción escolar y marginalidad, sistema de evaluación institucional, decreto 1290

\begin{abstract}
This reflection arises from the conception of evolution analysis that is presented in the curriculum and the evaluative practices of Educational Institution Santo Tomás de Aquino teachers. The methodology that guided the work was documentary. The conclusions were approached under the interpretation of Decree 1290, which showed that: 1 . There is a distance between the way the institutional evaluation system is organized against the evaluation criteria; 2 . The pedagogical and evaluative practices of many teachers differ from the approach proposed by Decree 1290, and in addition to this, they differ from the same SIE that they themselves have built as an institution; 3. In the prescribed documents and pedagogical practices of the teachers underlies a technicist conception of the evaluation; 4. Evaluation should be an instrument that promotes knowledge, not reprobation, desertion, social exclusion and marginalization.
\end{abstract}

Keywords: evaluative practices, school desertion and marginality, institutional evaluation system, decree 1290. 


\section{Introducción}

n Colombia, a partir de la Ley 115 de 1994, que es la Ley General de $\checkmark$ Educación para la Educación Regular, y el Decreto 1860 que reglamenta lesta ley, se le otorgan autonomía a las instituciones educativas (en adelante, IE) para que organicen sus sistemas de evaluación institucional, siguiendo algunos parámetros generales que establece el Decreto 1290 de 2009, el cual entre otras cosas reglamenta la evaluación del aprendizaje y promoción de los estudiantes en los diferentes niveles de la educación regular.

Actualmente se observa a través del mismo ejercicio docente que, la autonomía de algunas IE no está siendo aprovechada en su totalidad, e incluso, se ha generado una errónea interpretación del Decreto 1290, porque muchos sistemas de evaluación institucionales son una colcha de retazo que carece de criterios claros en la evaluación. También las prácticas evaluativas de algunos docentes dejan mucho que decir, dado que en ocasiones no están bien fundamentadas, y peor aún, difieren del sistema de evaluación que ellos mismos han confeccionado.

Lo anterior, no es ajeno al caso de la Institución Educativa Santo Tomás de Aquino del municipio de Titiribí, Antioquia, de donde parte esta reflexión crítica, la cual tiene como propósito: a) analizar las concepciones que sobre evaluación tiene la institución objeto de estudio, a partir de su Sistema de Evaluación Institucional, el Decreto 1290 de 2009 y las prácticas evaluativas de algunos docentes; b) reflexionar sobre el concepto de evaluación como un instrumento o herramienta vinculado a la enseñanza y el aprendizaje, que permita pensar en otras formas de evaluar más allá del tecnicismo; c) fundamentar las prácticas pedagógicas para recuperar el rol docente; d ) presentar una concepción de la evaluación alejada del castigo, la exclusión, la deserción y la marginalidad.

Por consiguiente, el abordaje de estas líneas que se enfocan en un artículo de reflexión, recoge un estudio de corte documental durante seis meses, el cual se abordará de manera resumida de la siguiente forma: primero, se contextualizan algunos caracteres de la escisión-problemática que existe entre el SIE, el Decreto 1290 y las prácticas educativas en la institución objeto de estudio; segundo, se presentarán las concepciones de evaluación que subyacen en algunos docentes y el SIE de la institución; tercero, se reflexiona sobre la evaluación como herramienta de conocimiento. 


\section{Desarrollo del tema}

La evaluación y las prácticas pedagógicas en la IE Santo Tomás De Aquino a la luz del decreto 1290

i bien es cierto que la evaluación tiene muchas concepciones, por $\circlearrowleft$ ejemplo, Botello (2012) entiende por evaluación cualquier forma que permita al docente apreciar el grado de cumplimiento de un objetivo, meta o tarea, y Jornet, (2012) sostiene que la evaluación debe mediar la relación docente y estudiante. Para el presente manuscrito, se entiende la evaluación como una herramienta de conocimiento que permite reorientar el proceso de enseñanza y aprendizaje. Bajo esta concepción se hizo en análisis al SIE de la IE Santo Tomás de Aquino, donde se pudo evidenciar que este cumple con los requisitos de forma más no de fondo que establece en el Decreto 1290 de 2009; pero difiere sustancialmente en las concepciones frente a la evaluación.

Por ejemplo, el Decreto 1290 en su artículo 3, señala que las IE deben indicar en el SIE, los propósitos de la evaluación institucional de los estudiantes; el artículo 4, habla que las IE deben definir el sistema institucional de evaluación de los estudiantes; el 6, indica que debe existir un proceso de promoción escolar claro y coherente; el 8, establece unas pautas generales para la creación del sistema de evaluación institucional de los estudiantes; el 11, señala las responsabilidades del establecimiento educativo sobre los procesos evaluativos; el 12, habla sobre los derechos del estudiante frente a la evaluación; el 14, expresa la importancia de la participación de los padres de familia en el proceso evaluativo.

Aunque todos estos requisitos están ordenados y prescriptos en el documento-SIE, hay una diferencia subyacente: existe una mala interpretación del Decreto 1290, y ello, conlleva a una errónea aplicación del mismo. Eso se puede otear con tan solo analizar los artículos 3 y 4 que arriban se citan.

De hecho, frente al artículo 3: Propósitos de la evaluación institucional, se puede decir que, la IE Santo Tomás de Aquino, atiende a lo que ordena este artículo, pero de forma, más no de fondo, dado que ha elegido 12 propósitos que debieran orientar la forma de evaluar pero en el ejercicio docente esto no se aplica. Por ejemplo, en uno de sus propósitos sostiene que la evaluación es "continua, porque permite asumir la evaluación como un proceso permanente 
que posibilita reorientar la formación de los educandos" (IE Santo Tomás de Aquino, 2015, P. 12).

No obstante, se observa que la mayoría de los docentes no hacen la evaluación continua para reorientar los procesos. Lo que comúnmente se realiza es la toma de calificaciones constantemente mediante un proceso lineal y no retrospectivo para hallar las dificultades en el proceso de enseñanza y aprendizaje.

Ahora bien, revisando el Decreto 1290 se evidencia que hay autonomía para las IE, de tal forma que estas puedan organizar sus propósitos de evaluación, para que las acciones de los docentes y administrativos sean coherentes con esos propósitos que ellos mismos han validado y construido, pero tampoco se hace. Docentes y directivos parecieran que entendieran autonomía "como hacer lo que quiera de manera independiente", en tanto que: 1) la hechura SIE no se ajusta a lo ordenado en el Decreto 1290. Precisamente, se hace lo contrario de lo esté propone en cuanto a los propósitos que deben guiar la evaluación institucional; y 2) cada profesor evalúa como lo desea sin tener en cuenta los parámetros que fija el SIE. De hecho, existen actas del Comité Académico Institucional que orienta la manera cómo se deben evaluar a los estudiantes con sus respectivas rúbricas, y no son tenidas en cuenta por la mayoría de los maestros; $y$, los directivos tampoco velan para que tales orientaciones tengan cumplimiento.

Lo mismo sucede con la interpretación del artículo 4: Definición del sistema institucional de evaluación de los estudiantes, en él, el inciso 3 dice que las IE tienen la obligación de propiciar estrategias de valoración integral de los desempeños de los estudiantes; el inciso 6, apostilla que se deben crear estrategias de apoyo necesarias para resolver situaciones pedagógicas pendientes de los estudiantes; el inciso 5 dice que deben existir procesos de autoevaluación de los estudiantes; y el inciso 1 indica que se deben fijar los criterios de evaluación y promoción.

Como se puede observar, en el Decreto 1290, subyace una concepción bastante cualitativa de la evaluación. Pero es lamentable que, frente al inciso 3 y el inciso 6 del artículo $4^{\circ}$, muchos docentes en las IE como la que se cita, no propicien estrategias para la valoración integral de los aprendizajes, ni creen estrategias de apoyo necesarias para resolver situaciones pedagógicas pendientes de los escolares, dado que sus miradas únicamente se fijan en la cuantificación de una valoración. Esto es 
preocupante, teniendo en cuenta que la función principal de un maestro es formar y educar mediante la enseñanza, no rajar o reprobar a los estudiantes. En tal sentido, es muy lamentable que muchos docentes nieguen los procesos de recuperación o simplemente no los hagan por simple capricho como ha sucedido y sucede en esta importante IE. Esto es grave en tanto que, primero, el docente no está cumpliendo con legislación en materia de evaluación; y segundo, no se está preocupando por darle la oportunidad a aquellos estudiantes que necesitan de su ayuda u orientación. Lo importante aquí no es la nota por la nota, sino la oportunidad que se brinda al educando que tienen dificultades mediante un plan de recuperación, porque en un proceso de evaluación lo relevante no es la nota, sino el aprendizaje de los estudiantes.

No se equivoca Larraguivel (1998) cuando al criticar algunas formas de evaluar sostiene que, las estrategias evaluativas más comúnmente usadas suelen centrarse, ya sea en la medición del rendimiento escolar o en el empleo de estadísticas escolares. Lo anterior genera enormes peligros, tal como lo afirma Sanjurjo y Vera (1994) refriéndose a los efectos psicosociales que tiene la evaluación: "A veces los docentes abusamos de una cierta manía etiquetadora, y lo que es más grave, actuamos como si fuera nuestra única función. Una vez que hemos dictado que un alumno no es bueno o es sobresaliente nos sentamos a esperar que la naturaleza haga lo suyo" (P. 128).

Un SIE enfocado en la nota pareciera que olvidara la responsabilidad social de la escuela: formar para la vida, pero también, luchar contra la exclusión, la marginalidad. Siguiendo a Sanjurjo y Vera (1994), se puede inferir que, la escuela o su SIE, lucha contra la exclusión cuando sin dejar de cumplir su función de acreditador/a, deja de actuar como ente seleccionador, valorando únicamente la supervivencia del más apto, aquel que por sus conocimientos innatos obtenga las calificaciones o exámenes más altos y abandonado a los que carecen del innatismo, que en términos de Bourdieu y Passeron (2014) serían los que no tienen capital cultural.

Además, la escuela o su SIE luchan contra la marginalidad cuando los docentes y administrativos son conscientes que la evaluación tiene consecuencias y connotaciones diferentes. Consecuencias, que muy bien las define Sanjurjo y Vera (1994), como connotaciones: ideológicas, sociales, psicológicas, pedagógicas y técnicas. 
Tiene connotaciones ideológicas porque según estas autoras, las prácticas que se vivencian en la escuela, son producto de la influencia que ha ejercido en ella el contexto social, principalmente sobre las formas de evaluar, que se enmarca en las concepciones históricas sobre el evaluar, bajo la ejemplificación de creer y defender la selección natural o innatismo mediante las prácticas evaluativas donde sobrevive el más fuerte.

Para Díaz Barriga (1992), es un error defender la selección natural, porque esto lo que hace es fomentar la injusticia social como lo hace la invención de las pruebas de inteligencia que constituyó una visión equivocada para justificar las diferencias sociales, presentándolas solo como individuales, apoyándose en el concepto de coeficiente intelectual. Estos según este autor "[.... redujo el problema de la injusticia social a una dimensión biologista. La sociedad queda liberada de los problemas éticos que crean la injusticia cuando puede mostrar que las diferencias sociales son únicamente el resultado de diferencias biológicas" (p.41). En efecto están en un craso error, aquellos que piensan que la exclusión social de unos sujetos, en cierta manera es responsabilidad de ellos mismos, o mejor, su lugar en la sociedad dependía de su constitución biológica intelectual. Si es bueno le irá bien y será un buen estudiante con altas cualidades, pero si no tiene altas capaciades y cualidades intrísecas, le irá mal. Lo insólito es que en muchas ocasiones es la evaluación mal concebida la que determina esto.

Por otra parte, las connotaciones sociales de la evaluación tiene que ver con el fracaso escolar, es decir, dependiendo de la concepción de educación que tenga una escuela, así será su forma de evaluar y de aquellas prácticas evaluativas puede depender el fracaso escolar que automáticamente pueden incidir en el proyecto de vida de los alumnos, pues cuando existe una tasa muy alta de reprobación, aparece la deserción y con ello la marginalidad.

Particularmente en la IE analizada, de acuerdo a datos que tiene sobre la repitencia, en el año 2016 se presentó un 15\% de deserción escolar la cual fue argumentada por los estudiantes como causa de las complejas y memorísticas evaluaciones de algunos docentes que impedían que los estudiantes tuvieran posibilidades de recuperarse. Y lógico, una vez el estudiante siente que numéricamente no tiene condiciones, su decisión es desertar.

De igual forma, se evidenciaron otras causas como la baja condición económica de las familias, lo que hace que el estudiante abandone la escuela o colegio 
para irse a trabajar en los cafetales de la zona, e incluso algunos ingresan a las bandas criminales como fuente de obtención de ingresos, esto, sin contar los estudiantes que no tienen condiciones económicas para transporte escolar, dado que la Jornada Única Escolar, que tiene la obligación de proveerle transporte, no lo hace o lo hace de manera discontinua por los poco recursos que el Ministerio de Educación Nacional gira a la gobernación, y esta al municipio. Todo ello provoca la marginalidad escolar.

Vale recordar que las teorías de la marginalidad se clasifica en dos grupos: las teorías que entienden que la educación es un instrumento de igualdad social, por lo tanto, de superación de la marginalidad y la teoría que entiende que la educación es un instrumento de discriminación social, luego, un factor de marginación (Saviani, 2017).

En consecuencia, cuando un estudiante es considerado torpe, bruto, no apto para ser promovido, verdaderamente él se lo cree. Ahora, como efectivamente se lo cree, muchas veces se gesta en él una fobia a la escuela y deserta tal cual lo indican las estadísticas en la IE.

Las connotaciones pedagógicas de la evaluación, tienen que ver con la permeabilidad de vicios que crean un tipo de subjetividad en los estudiantes. Esto, dependiendo de la concepción que tengan los estudiantes y docentes sobre la evaluación, dado que si la práctica evaluativa del docente es corroborar datos, los estudiantes aprenderán datos aunque en el currículum figuren otros objetivos (Sanjurjo y Vera, 1994), porque los estudiantes internalizan muchas de las prácticas de sus maestros.

En rigor, si un docente evalúa resultados en objetivos solamente, el estudiante en últimas, termina respondiendo solo a los objetivos trazados por el profesor para ser acreditado; situación que tiene consecuencias en la vida del docente, porque: "el docente quedará atrapado en una gran maquinaria, como autor rutinario de atribuir calificaciones, e inconscientemente muchas veces utilizará la evaluación como refugio para salvar su prestigio, su lugar de poder y su autoridad" (Sanjurjo y Vera, 1994, P. 129).

De igual forma, las connotaciones técnicas tienen que ver, con validar instrumentos y criterios de evaluación que sean conocidos por todos los actores educativos, porque según Sanjurjo y Vera (1994), esto permite enriquecer y no empobrecer el proceso, pues, al democratizar los instrumentos 
y criterios, permiten reflejar y comprender los procesos que se llevan a cabo, lo que posibilita que los juicios sean más fundamentados. Sin embargo, pese a la importancia de realizar esta labor, las prácticas evaluativas en la IE no se presentan de forma óptima, pues al estudiante no se le comparten los criterios de evaluación que serán tenidos en cuenta y mucho menos una rúbrica que le permita empoderarse y hacer parte del proceso de evaluación como autoaprendizaje activo.

En consecuencia, es una invitación para reflexionar sobre los instrumentos, su finalidad y las metodologías de evaluación. Concebir los instrumentos así, de manera democratizadora, es distanciarse en la práctica del positivismo pedagógico que cree hay formas de evaluar mejores que otras y de la objetividad científica pura a la hora de evaluar. Precisamente, Celman (1998) como no cree en tal objetividad científica sostiene que las pruebas con carácter de objetividad pura, fueron interrogadas porque: 1) hacían una fragmentación de los contenidos al dividir un tema o cuestiones en un gran número de ítems para resolver; 2) en ciencias sociales no se hacen planteamientos ni formulaciones cerradas, en opciones excluyentes; 3) Solo se evalúan los resultados finales, pues no se evalúa el proceso. No obstante, esta autora hace más fuerte su crítica al respecto cuando afirma:

En algunas épocas de la historia de la evaluación educativa se creyó que existían formas de evaluación que eran indudablemente superiores a otras. Es decir, se ponía el acento en la manera de construir, de confeccionar el/los instrumentos con los cuales se procedería a evaluar, imponiendo especial énfasis en ciertos atributos que deberían de reunir dichos instrumentos. Con ello se pretendió diferenciar la evaluación "científica" de aquellas más ingenuas o intuitivas (p. 43).

Lo anterior, también tiene eco en las palabras de Alvares (1995) respeto al consenso social, donde muchos creen que la evaluación no puede ser de otro modo, cuando sostiene que pensar una forma de evaluar, sin duda tiene que ver con que "se crea así una ilusión de consenso social respecto a los objetivos, los usos y las funciones de la evaluación, hasta el punto en que el mismo consenso nos hace pensar y ver que la evaluación no puede ser de otro modo [...]" (174). Nótese que el tema de la evaluación ha tenido gran relevancia y ha sido objeto de análisis desde varios años atrás, sin embargo el desconocimiento de los docentes y de la comunidad en general 
va acompañado de las mismas prácticas evaluativas que han impedido el avance en la metodología educativa actual.

Tener una concepción así de la evaluación, es tener una visión de recorte de las realidades que nos arrojan los instrumentos de evaluación, pues se recorta, se cercena todo lo que no quepa en la visión objetivadora. Metafóricamente hablando, sería actuar como Procusto en la metodología griega del Lecho de Procusto que nos habla González (2014), donde este siendo hijo de Poseidón tenía una morada-casa, en la cual recibía a todos aquellos que buscaban refugio y alojamiento y una vez instalados, los obligaba a acostarse sobre un oxidadolecho. Si el cuerpo era muy largo, cercenaba sus extremidades, hasta que el tamaño del cuerpo se adecuara al lecho.

Con lo anterior, se quiere sugerir que: a) en la visión positivista de la evaluación, se mutila todo lo que no esté dentro de esa racionalidad; b) Las prácticas evaluativas cercenadoras, al cual solo le importa el resultado en términos numéricos, desconoce el proceso, lo ignora, porque lo que se trata es de diseñar instrumentos que sólo capten y se adecúen a su misma racionalidad. Exactamente, eso en lo que hace el Lecho de Procusto, lo que no está dentro de su medida, lo elimina.

\section{Prácticas pedagógicas vs deserción escolar}

or otra parte, se pudo identificar en el análisis que se realizó al SIE de la IE Santo Tomás, que en este centro se trabajan las competencias del

_ saber, saber hacer y ser. El saber tiene que ver con los resultados de los exámenes, que equivale a un 35\%; el saber hacer con los trabajos, consultas y talleres, que cuantifican un 35; el ser con el comportamiento de los estudiantes, equivale a un 20\%); y la autoevaluación un 10\%. Lo preocupante es que la categoría ser, hay docentes que la toman como poder y medio de castigo con algunos alumnos. Es decir, si el estudiante es muy hiperactivo, habla, se mueve, se ríe, camina, toca a un compañero, el docente en esta categoría lo evalúa con una baja calificación aduciendo un mal comportamiento. Cosa que debiera ser lo contrario, aprovechar esos acontecimientos (hiperactividad, habla, movimientos, risa), para interrogarlos y revisar qué pasa tanto en su clase como en el proceso de enseñanza y aprendizaje.

Lo grave es que la IE en cuestión, en su Manual de convivencia (2014), concretamente en su artículo 72: Tipos de sanciones, estipula que 
los estudiantes que reincidan en un comportamiento inadecuado serán expulsados hasta por 8 días, cuando afirma: "La suspensión temporal de las actividades académicas y extracurriculares, de uno a 8 días, será por presentar inadaptación a las normas de la institución" (p 39), contemplando la expulsión definitiva de los estudiantes que reprueban el año por tres veces consecutivas.

La concepción de evaluación que subyace tanto en los documentos institucionales (SIE, manuales de convivencia), y las prácticas pedagógicas de algunos docentes, se evidencia en la realidad que refleja la siguiente tabla, frente a la evaluación de los aprendizajes.

\section{Relación de matrícula inicial y final vs aprobación y deserción}

Tabla. Grado de repitencia y deserción en la IE Santo Tomás de Aquino año 2015.

\begin{tabular}{|c|c|c|c|c|c|c|c|c|}
\hline Grados & $\begin{array}{c}\text { Matrícula } \\
\text { inicial }\end{array}$ & $\begin{array}{l}\text { Matrícula } \\
\text { Final }\end{array}$ & $\begin{array}{l}\text { Estudiantes } \\
\text { Aprobados }\end{array}$ & $\begin{array}{l}\text { Estudiantes } \\
\text { reprobados }\end{array}$ & $\begin{array}{l}\text { Estudiantes } \\
\text { desertores }\end{array}$ & $\begin{array}{l}\text { Estudiantes } \\
\text { pendientes }\end{array}$ & $\begin{array}{c}\text { Estudiantes } \\
\text { cancelados }\end{array}$ & $\begin{array}{c}\text { Fuga de } \\
\text { estudiantes }\end{array}$ \\
\hline $1^{\circ}$ & 67 & 59 & 47 & 10 & 2 & 2 & 6 & 8 \\
\hline $2^{\circ}$ & 58 & 51 & 39 & 8 & 2 & 4 & 5 & 7 \\
\hline $3^{\circ}$ & 92 & 83 & 46 & 19 & 3 & 18 & 6 & 9 \\
\hline $4^{o}$ & 80 & 74 & 56 & 9 & 4 & 9 & 2 & 6 \\
\hline $5^{\circ}$ & 72 & 64 & 48 & 9 & 4 & 7 & 4 & 8 \\
\hline $6^{0}$ & 163 & 127 & 71 & 28 & 23 & 28 & 13 & 36 \\
\hline $7^{\circ}$ & 100 & 76 & 39 & 17 & 13 & 20 & 11 & 24 \\
\hline $8^{\circ}$ & 113 & 93 & 52 & 22 & 10 & 19 & 10 & 20 \\
\hline $9^{\circ}$ & 75 & 70 & 39 & 13 & 5 & 18 & 0 & 5 \\
\hline $10^{\circ}$ & 64 & 53 & 44 & 2 & 4 & 7 & 7 & 11 \\
\hline $11^{\circ}$ & 69 & 67 & 62 & 5 & 2 & 0 & 0 & 2 \\
\hline Total & 953 & 817 & 543 & 142 & 72 & 132 & 64 & 136 \\
\hline
\end{tabular}

Fuente: Autores (2015), que retoman de las Actas de Evaluación y Promoción

Obsérvese que entre las columnas de deserción y estudiantes cancelados, se produce una fuga de alumnos de un total de 136, que muy seguramente sin desconocer otros factores sociales, van a aumentar la exclusión y la marginalidad; cifra que puede aumentar, si se detiene la mirada en la columna de estudiantes pendientes, pues de los 132 que quedaron pendiente para resolver su situación academia para enero de 2016, muchos perdieron las recuperaciones, y por consiguiente el año. Conociendo los antecedentes de estudiantes, muchos de ellos abandoran la escuela. 
Así mismo preocupa que de los 953 estudiantes que iniciaron el año, solo 817 pudieron terminarlo; pero de esos 817, 274 que corresponden al 33\% que finalizaron el año de manera accidentada muestran que: 1. reprobaron; 2. desertaron, 3. quedaron pendiente para enero del año siguiente; y 4. cancelaron la matrícula, logrando culminar satisfactoriamente solo el $67 \%$ que corresponde a 543 estudiantes.

De otro lado, en lo ateniente al inciso 1, el proceso de promoción escolar de la institución educativa en mención, aunque tiene contemplado en el SIE los procesos de autoevaluación de los estudiantes, este no se hace correctamente, porque, primero, no se han fijado unos criterios de autoevaluación; pero además, cuando los estudiantes se autoevalúan, los docentes no aceptan dichas valoraciones, lo que evidencia la necesidad de construir unos criterios mínimos. En este sentido se comprenden las palabras de Di Francesco (2005), cuando sostiene que:

Las prácticas evaluativas contienen la presencia de dos sujetos, uno que da y el otro que demanda, cuya relación está sostenida por el conocimiento. Para que esta relación sea transparente es importante definir las reglas de regulación que existan. Y en este análisis podemos preguntarnos ¿cómo se establecen las reglas de la evaluación en la práctica educativa? ¿Desde el interior de las instituciones, de los docentes o desde parámetros externos de control ya que están establecidos? (p. 37).

Es así como la autoevaluación de los estudiantes está concebida como un listado de indicadores de desempeño, Fernández y Vanga (2015) donde el mismo estudiante internaliza su propia formación estructurada del conocimiento adquirido, dando paso a que el docente identifique por medio de ella la reflexión cuantitativa. Esto va en detrimento con la autoevaluación estudiantil de la IE analizada, dado que el estudiante valora su formación en un puntaje de 1 a 5 , siendo esta a su vez permeada por el acompañamiento valorativo del docente, quien le otorga una calificación a su modo, desconociéndose los criterios que le conducen a promediar alto o bajo la autoevaluación del mismo estudiante.

En consecuencia, validar unas reglas de regulación para la autoevaluación, es fundamental, no solo para evitar conflictos, sino para saber qué y para qué se evalúa y a partir de ahí, buscar la mejora de la enseñanza, del aprendizaje. 


\section{Concepciones de la evaluación de los docentes en la IE Santo Tomás} de Aquino a partir del SIE frente al del Decreto 1290

partir de la situación antes descrita, se puede inferir que el SIE
y las prácticas pedagógicas de algunos profesores de la IE Santo
Tomás de Aquino, cabalgan en una concepción empírico analítica o mecanicista, pues solo se enfocan en los resultados y descuidan el proceso. Se puede entender además que, toman la evaluación cómo algo separado del proceso de enseñanza y aprendizaje cuando realmente entre estos tres momentos (enseñanza, aprendizaje y evaluación), no debe existir separación, sino integralidad y complementariedad. Vale la pena traer a colación las palabras de Celman (1998), cuando resaltando el principio de integralidad del enseñar, evaluar y aprender sostiene que, la evaluación no es un apéndice de la enseñanza y del aprendizaje, sino que es parte de los dos, hasta el punto de afirmar de manera categórica que:

Con este principio se pretende sacar la evaluación del lugar en el que comúnmente se la ubica: un acto final desprendido de las acciones propias de la enseñanza y el aprendizaje. Se opone a adjudicar a la evaluación el papel de comprobación, de constatación, de verificación, de unos objetivos y unos contenidos que deben por medio de pruebas, exámenes, ser sometidos al acto de control que permita establecer el grado en que los han incorporado (p. 37).

Si a la evaluación se le da su estatus de un momento que es integrado a la enseñanza y el aprendizaje, se convierte en una poderosa herramienta de conocimiento que: 1) mejora los procesos educativos mediante las búsquedas de nuevas formas de enseñar y de aprender; 2) el docente en la escuela ocupa su rol de sujeto pedagógico.

Respecto a lo primero es indubitable que darle un estatus a la evaluación así, permite emplear otras prácticas pedagógicas, diferentes a las validadas por la educación tradicional, donde el docente muchas veces evalúa de forma mecánica sin reflexionar sobre su oficio, la enseñanza, y el aprendizaje de los escolares.

No en vano dice Mosquera (2015) que unas de las mejores armas que tiene el docente es la reflexión pedagógica para mejorar la práctica docente; posición que también defiende Perrenoup (2007), al apostillar que la práctica reflexiva es fundamental en el oficio de enseñar. En este caso, la evaluación es una 
herramienta que a través de sus resultados, da insumos para la reflexión pedagógica que permita deslizar la mirada sobre la enseñanza y el aprendizaje. Por tanto, se corrobora, que los tres momentos: enseñanza, aprendizaje y evaluación son inseparables, y no debe presentarse escisiones entre ellos.

Respeto a lo segundo, si a la evaluación se le da su estatus, el maestro en la escuela retoma su rol de sujeto pedagógico, hurtado por el tecnicismo, porque al reflexionar sobre la realidad que la evaluación le arroja, no "quejándose", sino en buscar comprender la falla, para de esta manera mejorar lo que se requiera.

Esto por un lado, por otro, como el docente ha perdido su poder de sujeto pedagógico, dado que muchas veces cede su rol al enfoque mecanicista, dedicándose a solo seguir instrucciones dadas por otros, los llamados "expertos o especialistas". Hecho grave, porque según Celman (1998): "Un enfoque prescriptivo de la educación supone que el docente, limita sus trabajo a ejecutar en el aula las indicaciones que otros han confeccionado... Esta suposición no sólo es éticamente insostenible sino, también, empíricamente falsa" (p38).

En conclusión, la evaluación es herramienta de conocimiento, en tanto que sirva para reflexionar sobre la enseñanza y el aprendizaje. Pero también, es uno de los instrumentos que dependiendo de la región de conocimiento o perspectivas con los cuales los docentes miren la realidad, puede ayudar a que el maestro recupere su rol de sujeto pedagógico al repensar por sí mismo la educación y no esperar que los especialistas y las editoriales lo hagan por él. Y esto lo puede hacer con los insumos o información que arroja la evaluación.

\section{¿Qué viene siendo entonces el acto de evaluar?}

¿Por qué debemos preguntarnos sobre el saber y la educación cuando indagamos sobre las concepciones de la evaluación? Porque en el marco de la Educación y el saber, como campo de análisis, es desde donde interpretamos las representaciones que se construyen sobre la evaluación (Di Francesco, 2005, p. 34).

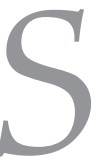
e sigue argumentado que la evaluación en las instituciones educativas, antes de ser un instrumento seleccionador (unos buenos y otros malos, unos competentes y otros incompetentes); no deja de ser un 
instrumento de castigo, porque quien la aplica tiene el poder; un instrumento de exclusión que motive a la deserción y marginalidad, que causa laceraciones psicológicas por la mortificación que sufre quién pierde un examen al quedar etiquetado. Esta debe ser un instrumento y herramienta de conocimiento, tanto para los docentes, directivos, estudiantes y padres de familia, dependiendo de la concepción que se tenga de la misma que permita una realimentación constante en el ejercicio pedagógico.

La evaluación puede ser una herramienta de conocimiento para el docente, en la medida

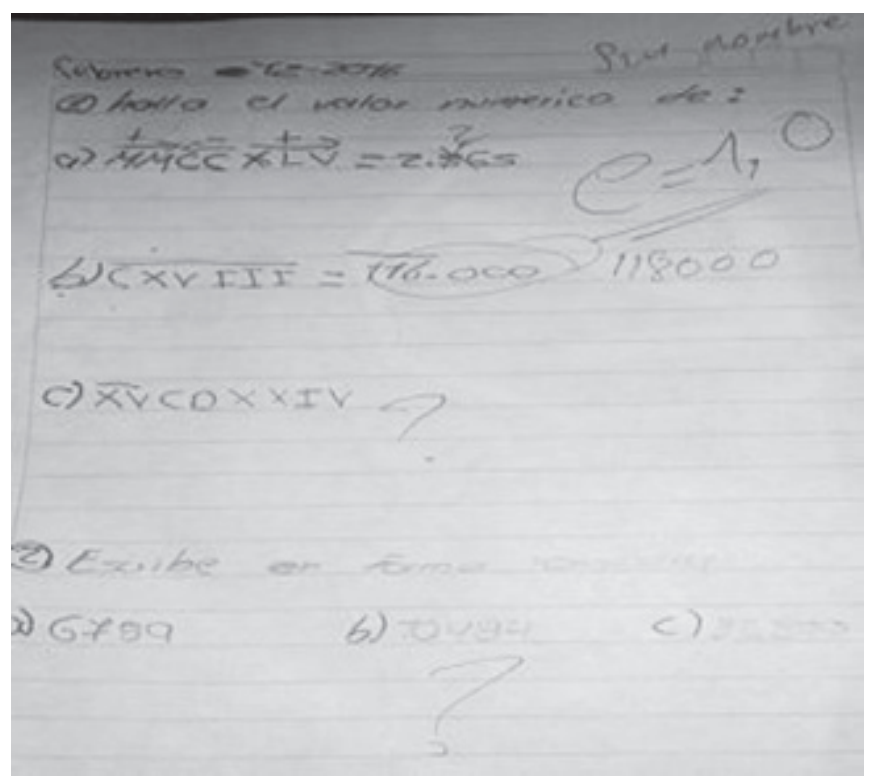

Foto. Examen de matemática, grado séptimo IE Santo Tomás de Aquino

Fuente: Carlos Enrique Mosquera (2016) en que le permita reflexionar para corregir y mejorar el proceso de enseñanza y aprendizaje, pues no solo buscará la manera de cómo los estudiantes aprenden mejor, sino que, al mismo tiempo él aprende a aprender a cómo enseñar mejor.

Sería cómo una especie de tematización que el docente hace de sí mismo para mejorar la práctica pedagógica, pues tal como afirma Foucault (2012), jamás podrá haber una transformación de sí (sujeto), si no existe un conocimiento previo de sí. Para este caso, requiere que el docente examine sus prácticas evaluativas y las tematice. Es decir, si el docente no se conoce a sí mismo tematizando y fortaleciendo aquello que deba mejorar dentro de su oficio como sujeto pedagógico, será muy difícil que piense en otras formas de enseñar, de aprender y de evaluar.

La evaluación para este caso es una herramienta que posibilita que el docente deslice la mirada sobre su práctica, siempre y cuando interrogue los resultados evaluativos; pues no olvidemos que según Díaz Barriga (1990), citado por Sanjurjo y Vera, (1994) la realidad que muestran los exámenes es consecuencia de lo que sucede en el aula, y que el cambio fundamental debe realizarse en las estrategias metodológicas que emplee el maestro. El mismo Comenio, retomado por Díaz Barriga (1992) ya lo había dicho mucho tiempo atrás cuando recomienda que en el momento en el que el alumno no aprende, debe revisar su método y que no se debe castigar porque se le 
crearía una aversión sobre el estudio, lo cual en la actualidad se ve reflejado en el proceso educativo.

Lo anterior dentro de otro ángulo de análisis, también, es un llamado para que el docente se sacuda un poco del tecnicismo, y deje de ser un mero ejecutor de formas homogeneizadas de enseñar y de evaluar. El maestro necesita recuperar su rol de sujeto pedagógico. Una de las formas de recuperarlo es sabiendo que: "todo currículo y cualquier metodología por organizado que estén, son sólo propuestas y sugerencias que se transforman por acciones mediadoras de las instituciones y los docentes" (Celman, 1998. p. 38).

Se sabe que el maestro debe cumplir unas tareas y someterse a una racionalidad legalista, administrativa, y en cierto modo hasta positivista, y que sobre todo debe evaluar para acreditar; pero ese no es el problema, el problema es cómo lo hace y para qué finalidad lo hace: ¿para enseñar? ¿Para aprender de los resultados mediante el análisis de los procesos? ¿Para reprobar? ¿Para demostrar poder y castigo? ¿Para seleccionar?, porque como dice Celman (1998):

Es muy diferente una evaluación destinada a comprobar que se ha retenido de un tema que se estudió consultando un texto único, que aquella otra destinada a conocer el tipo de relaciones que el estudiante sea capaz de hacer entre distintos actores, al interior de dicho tema y con otros, las opiniones que les merecen, las implicaciones a situaciones diferentes, las preguntas en las que se quedó pensando, etcétera (p. 45).

La evaluación puede ser una herramienta de conocimiento para los directivos, si por un lado, analizan y ayudan a orientar las prácticas de los docentes de acuerdo a la propuesta educativa que tienen validada en los Horizontes Institucionales o Imagen Objetivo, que entre otras cosas está guiada por un determinado modelo educativo.

En referencia a lo anterior, Salinas (1997) hace énfasis que un modelo educativo para la práctica o desde la práctica siempre representa una opción, no solo pedagógica, sino también ideológica, sobre el papel y las funciones de la escuela, y de nosotros mismos como docentes, con respecto a la sociedad, los alumnos y alumnas, el conocimiento, etcétera. Opción esta que indudablemente, también, es evaluativa, pues aunque se es consciente 
que existen muchas formas de evaluar según la concepción de educación que tenga cada sujeto, también, se sostiene que las practicas evaluativas no se pueden dar al azar, deben de fundamentarse y esto es posible desde una perspectiva de la educación, que a su vez, está orientada desde los modelos o referentes teóricos.

En otras palabras, lo que se propone es que la forma de evaluar, como en últimas, depende de la concepción de qué es educación para el maestro; que por lo menos, se respeten los criterios de evaluación que una determinada IE ha construido, y para ello, es necesario la gestión de los directivos. Gestión entendida no en perspectiva burocrática, sino como búsqueda de la mejora institucional, que debe apuntar siempre a responder tanto al macro currículo (normas, orientaciones del Ministerio de Educación Nacional), como al micro currículo (PEI). De hecho, los directivos en cierta medida son corresponsables, por los niveles de repitencia y deserción, si se interroga su capacidad de gestión para atacar el flagelo.

Esto se puede ver por ejemplo desde la racionalidad del Estado colombiano, si se analiza por un lado, el Decreto 325 de febrero de 2015, que establece el Día E (Día de la Excelencia Educativa), y este a su vez, crea el Índice Sintético de la Calidad Educativa (ISCE), que entre otras cosas, es una pésima mirada de la evaluación, porque premian con recursos económicos a las mejores IE que obtengan buenos resultados evaluativos y castigan a las que no los obtengan, mediante el remordimiento psicológico y señalamiento que poseen los paupérrimos resultados en pruebas estandarizadas, donde, tanto, los administrativos y los docentes son los principales blancos de críticas.

Por otro lado, y apartados de la racionalidad anterior, se sostiene que los directivos, son corresponsables de los niveles de repitencia y la deserción, dado que, las decisiones académicas que tomen en esta materia (determinaciones y orientaciones) pueden beneficiar o fomentar la selección natural (repoblación, por consiguiente deserción y marginalidad). Es decir, sus directrices pueden estar de acuerdo con el "sobreviva quien sea fuerte y perezca el más débil", o puede liderar proceso junto con los docentes y padres de familia, donde se diseñen estrategias para favorecer a aquellos escolares que tiene menor capital cultural. De allí que los resultados que arrojan las evaluaciones institucionales son el insumo para ello.

La evaluación puede ser una herramienta de conocimiento para los estudiantes, siempre y cuando sea vista como un instrumento para reflexionar sobre su 
aprendizaje; cuando por ejemplo, el docente y los estudiantes analizan los resultados evaluativos juntos y son capaces de hallar las dificultades para corregirlas. Diferente es si se evalúa de un pantallazo a último momento para obtener una calificación, para castigar o para evaluar contenido. Esto si sería un hecho preocupante, porque, la evaluación dependiendo de su utilización, puede crear un tipo de subjetividad en los escolares.

Si se evalúa para que el estudiante aprenda, se generará una familiaridad con la evaluación. Si se evalúa para verificar contenido, el estudiante responderá a ese estímulo de manera memorista y mecánica. Si se evalúa para obtener una calificación, el estudiante estudia para notas altas y no con el deseo de aprender. Finalmente, si se evalúa para castigar, el alumno le teme a la evaluación y tendrá un doble comportamiento, bien cuando el docente no está al frente y mal cuando no esté, como si la formación recibida fuera para intervalos de momentos y no para la vida.

La evaluación puede ser una herramienta de conocimiento para los padres de familia, cuando les permite, primero, evidenciar los avances frente al aprendizajes de sus hijo; segundo, cuando en esos resultados puede mirar las dificultades que tiene sus hijos y buscan junto con los profesores formas para superar las dificultades encontradas. De ahí que la relevancia de la participación de los padres sea fundamental en los procesos evaluativos de los escolares, porque entre otras cosas, la formación y educación es también una acción mancomunada de la escuela y la familia.

\section{Conclusión}

Tr a evaluación en las instituciones educativas debe concebirse como herramienta de conocimiento integrada al proceso de enseñanza y de aprendizaje que permite: a) diseñar estrategias, metodología e instrumentos para mejorar los procesos de educabilidad, sobre todo, en los estudiantes que tienen dificultades en el aprendizaje; b) evitar la lejanía entre los criterios que establecen los sistemas de evaluaciones institucionales y las prácticas evaluativas de los docentes, donde el maestro asuma su rol de sujeto pedagógico, sujeto hacedor de la educación y de la cultura, que al utilizar los insumos de la evaluación los interrogue para repensar en otras formas de enseñar, de aprender y de evaluar; c ) reflexionar sobre las prácticas pedagógicas y evaluativas de muchos docentes, donde estos no conciban la evaluación como instrumento de castigo y de poder que promueva miedo en los escolares, la exclusión, la deserción y la marginalidad; d) democratizar los 
criterios de evaluación dentro de una comunidad educativa para ponerse de acuerdo en unos referentes mínimos que guíen las prácticas evaluativas; e) que los docentes puedan darse cuenta de las concepciones sobre evaluación que subyace en los documentos prescriptos, SIE y prácticas pedagógicas al reflexionar constantemente sobre ellos.

\section{REFERENCIAS BIBLIOGRÁFICAS}

Álvarez (1995). Volver a pesar la educación (Vol. II). Prácticas y discursos educativos (Congreso Internacional de Didáctica) España: Ediciones Morata, S.L, p. 174.

Botello, M. A. (2012). Evaluación, marginalidad y desarrollo. Reforma Siglo XXI , 19, 62-66.

Bourdieu, Pierre \& Passeron J. C. (2014). Los herederos. Los estudiantes y la cultura. Argentina Siglo XXI.

Celman, S. (1998). ¿Es posible mejorar la evaluación y transformarla en herramienta de conocimiento?: En Celman, S. (Coord) (1998). La evaluación de los aprendizajes en el debate didáctico contemporáneo. Buenos Aires: Paidós.

Di Francesco, A. (2005). Evaluación educativa. Representaciones de alumnos y docentes. Córdoba: Educando Ediciones.

Díaz Barriga, Á. (1992). Currículum y evaluación escolar. Buenos Aires: Rei Argentina, p.33.

Fernández, A. \& Vanga, M. G. (2015). Proceso de autoevaluación, coevaluación y heteroevaluación para caracterizar el comportamiento estudiantil y mejorar su desempeño. Revista San Gregorio, 7-13.

Foucault, M. (2012). Hermenéutica del sujeto, Argentina: Altamira.

Gonzáles, J. (2014). ¿Qué es la filosofía? La potencia de una pregunta imposible; Buenos Aires, Argentina: Biblos.

Jornet, J. (2012). Las dimensiones docentes y cohesión social: reflexiones desde la evaluación. Revista Iberoaméricana de Evaluación Educativa, 349-362.

Institución Educativa Santo Tomás de Aquino (2014). Manual de convivencia, p. 39.

Institución Educativa Santo Tomás de Aquino (2015). Sistema de Evaluación Institucional, P. 12.

Larraguivel, E. (1998). Propuesta de un modelo de evaluación curricular para el nivel superior. Una orientación cualitativa. México: Cuaderno del CESU.

Ministerio de Educación Nacional (Colombia) (2015). Decreto 0325. Recuperado de: http:// www.mineducacion.gov.co/cvn/1665/articles-349475_pdf.pdf 
Ministerio de Educación Nacional (2009). Decreto 1290 de 2009. Recuperado de: http://www. mineducacion.gov.co/1621/articles-187765_archivo_pdf_decreto_1290.pdf

Mosquera, C. E. (2015). Rumiar sobre el positivismo o transformar la práctica educativa pedagógica docente. Actualidades Pedagógicas, (65), 217-228. Recuperado de: http://revistas.lasalle. edu.co/index.php/ap/article/viewFile/2912/2771

Perrenoup, P. (2007). Desarrollar la práctica reflexiva en el oficio de enseñar, Madrid: Grao.

Salinas, D. (1997). Currículum, racionalidad y discurso didáctico. En Poggi , M.(Comp.) Apuntes y aportes para la gestión curricular. Buenos Aires: Kapelusz.

Fernández Sotelo, A. \& Vanga Arvelo, M. G. (2015). Proceso de autoevaluación, coevaluación y heteroevaluación para caracterizar el comportamiento estudiantil y mejorar su desempeño. Revista San Gregorio, 7-13.

Jornet, J. (2012). Las dimensiones docentes y cohesión social: reflexiones desde la evaluación. Revista Iberoaméricana de Evaluación Educativa, 349-362.

Saviani, D. (13 de abril de 2017). Las teorías de la educación y el problema de la marginalidad en América Latina. Revista Colombiana de Educación.

Sanjurjo, L. \& Vera, M. T. (1994). “Aprendizaje significativo y enseñanza en el nivel medio y superior, Buenos Aires, Ediciones Homo Sapiens. 

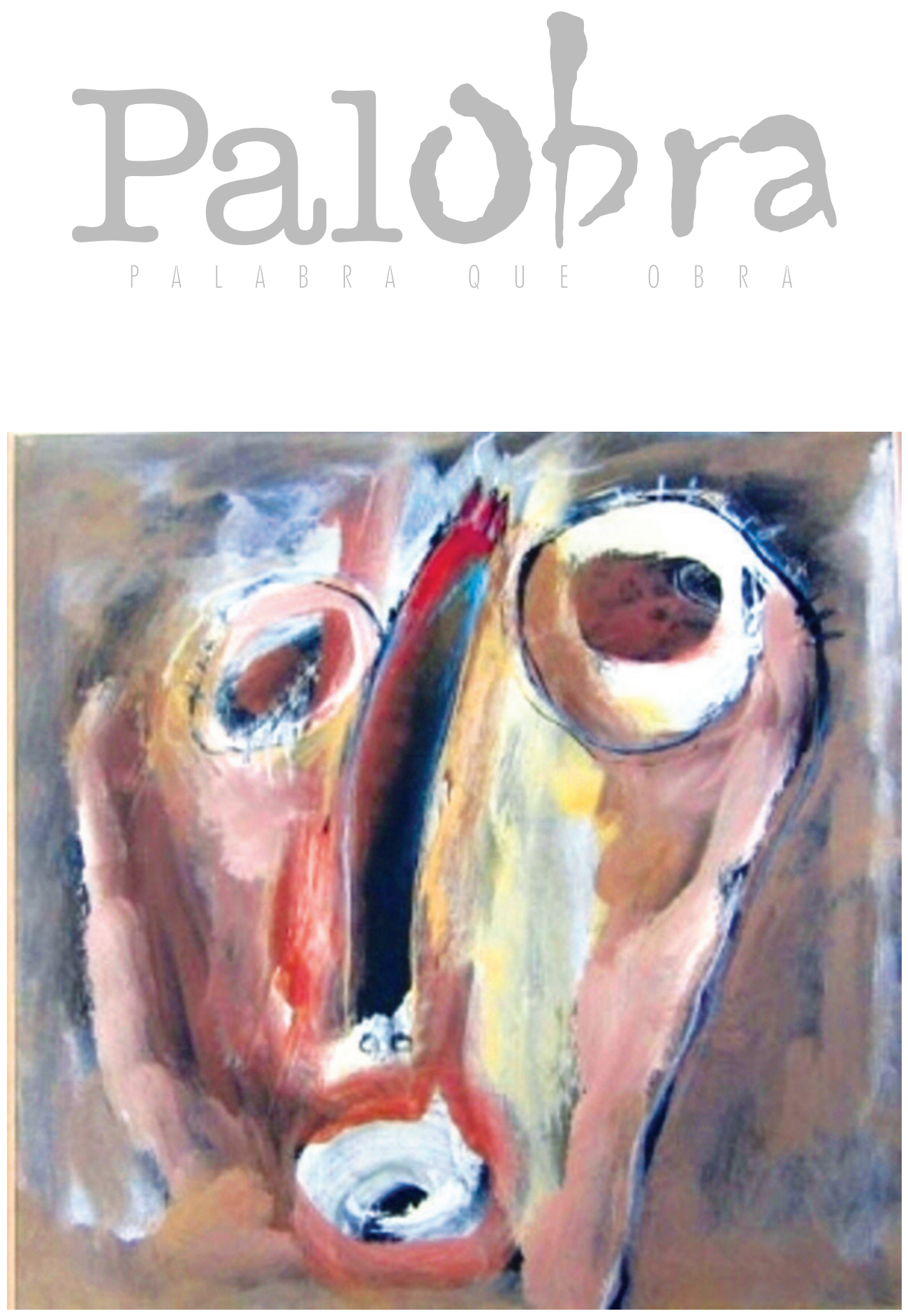

Obra pictórica de la serie "Rostros" de Eduardo Potrillé 